\title{
FINITE GROUPS AND INVARIANT SOLUTIONS TO ONE-DIMENSIONAL PLATEAU PROBLEMS
}

\author{
DAVID BINDSCHADLER
}

\begin{abstract}
Let $G$ be a finite group of isometries acting on a complete Riemannian manifold. Suppose that $B$ is a 0 -dimensional boundary which is $G$-invariant. If the order of $G$ divides the product of the cardinality of the orbit and the density of $B$ at each point, then a $G$-invariant absolutely length minimizing integral current with boundary $B$ can be constructed.
\end{abstract}

1. Introduction. Let $G$ be a finite group of isometries acting on a complete Riemannian manifold. Let $B$ denote a boundary consisting of a finite collection of points with integral densities. It is shown that if the order of the group divides the product of the cardinality of the orbit and the density of $B$ at each point, then there exists an invariant solution to the oriented Plateau problem. (That is, there exists an invariant collection of oriented length minimizing geodesics with boundary $B$ which minimizes total length among all collections of oriented curves with boundary $B$.) Known examples show that if the divisibility condition fails, there may exist boundaries with no invariant solution. (See [F1, 5.4.17].)

For other results concerning invariant solutions to the Plateau problem see $[\mathbf{L}]$, [B] and [BJ].

The author would like to thank the referee for suggesting the use of polyhedral chains instead of more abstract objects.

\section{Polyhedral 1-chains in a metric space.}

2.1. Definitions and notations. Let $(X, d)$ be a metric space.

A 0 -chain in $X$ is an integer valued function $\beta$ on $X$ such that $\{x: \beta(x) \neq 0\}$ is a finite set.

A polyhedral 1-chain (or 1-pchain) in $X$ is a nonnegative function $\alpha: X \times X \rightarrow Z$ such that $\{(x, y) \in X \times X: \alpha(x, y) \neq 0\}$ is finite and $\alpha(x, y) \alpha(y, x)=0$ for all $(x, y) \in X \times X$.

Let $\delta_{x}$ and $[x, y]$ denote the characteristic functions of $\{x\} \subset X$ and $\{(x, y)\} \subset$ $X \times X$, respectively. Then for any 0 -chain $\beta$ and 1-pchain $\alpha$, we have

$$
\beta=\sum_{x \in X} \beta(x) \delta_{x} \text { and } \alpha=\sum_{(x, y) \in X \times X} \alpha(x, y)[x, y]
$$

If $\beta$ is a 0 -chain and $\alpha$ is a 1-pchain, we define the mass of $\beta$, the mass of $\alpha$ and

Received by the editors August 27, 1979 and, in revised form, January 25, 1980.

AMS (MOS) subject classifications (1970). Primary 49F22; Secondary 49F20.

Key words and phrases. Plateau problem, area minimizing, integral current, invariant solution. 
the boundary of $\alpha$ by the equations

$$
\begin{aligned}
\mathbf{M}(\beta) & =\sum_{x \in X}|\beta(x)|, \\
\mathbf{M}(\alpha) & =\sum_{(x, y) \in X \times X} \alpha(x, y) d(x, y), \\
\partial \alpha & =\sum_{(x, y) \in X \times X} \alpha(x, y)\left(\delta_{y}-\delta_{x}\right)=\sum_{y \in X} \sum_{x \in X}(\alpha(x, y)-\alpha(y, x)) \delta_{y} .
\end{aligned}
$$

A cycle is a 1-pchain with zero boundary.

If $\alpha$ and $\gamma$ are two 1-pchains their chain sum is given by

$$
\alpha \oplus \gamma(x, y)=\max \{0, \alpha(x, y)-\alpha(y, x)+\gamma(x, y)-\gamma(y, x)\}
$$

for all $(x, y) \in X \times X$. The set of 1-pchains forms a commutative group under chain sums. Indeed, closure, existence of identity and commutativity are obvious. The inverse of $\alpha$ is the 1-pchain $\alpha$ given by $\Theta \alpha(x, y)=\alpha(y, x)$. And associativity follows from the equality $a=\max \{0, a\}-\max \{0,-a\}$. Also observe that

$$
\begin{aligned}
\alpha \oplus \gamma(x, y)+\alpha \oplus \gamma(y, x) & =|\alpha(x, y)-\alpha(y, x)+\gamma(x, y)-\gamma(y, x)|, \\
\partial \ominus \alpha & =-\partial \alpha \text { and } \partial(\alpha \oplus \gamma)=\partial \alpha \oplus \partial \gamma .
\end{aligned}
$$

A 1-pchain is called simple if it has the form $\sum_{i=1}^{l}\left[x_{i-1}, x_{i}\right]$, where $x_{i}=x_{j}$ implies $i=j$ or $\{i, j\}=\{0, l\}$.

In case $X$ were a complete Riemannian manifold and $T \in I_{1}(X)$ (see [F1, pp. $670,671])$ consisted of a sum of oriented length minimizing geodesic arcs with integral density, then one can associate with $T$ a 1-pchain $\alpha$ such that $\mathbf{M}(T)=$ $\mathbf{M}(\alpha)$ and $\partial \alpha$ is essentially $\partial T$. Also, given a 1-pchain $\alpha$, we can associate a sum of oriented length minimizing geodesic arcs with integral densities $T$ by associating with $\alpha(x, y)>0$ any length minimizing geodesic arc with boundary $\delta_{y}-\delta_{x}$ with density $\alpha(x, y)$. Then $T$ and $\alpha$ have the same boundary and $M(T)<M(\alpha)$. It is this connection that lets us exploit mass minimizing 1-pchain in the study of mass minimizing integral currents.

2.2. TheOREM. Given a polyhedral 1-chain $\alpha$, there exists simple 1-pchains $\sigma_{i}$, $i=1, \ldots, l$, such that $\alpha=\Theta_{i=1}^{l} \sigma_{i}=\sum_{i=1}^{l} \sigma_{i}, \mathbf{M}(\alpha)=\sum_{i=1}^{l} \mathbf{M}\left(\sigma_{i}\right)$, and $\mathbf{M}(\partial \alpha)=$ $\sum_{i=1}^{l} \mathbf{M}\left(\partial \sigma_{i}\right)$.

PROOF. By induction on the integer $\sum_{(x, y) \in X \times X} \alpha(x, y)$, it suffices to find a nonzero simple 1-pchain $\sigma$ so that

(i) $\alpha \ominus \sigma=\alpha-\sigma$,

(ii) $\mathbf{M}(\alpha)=\mathbf{M}(\alpha \ominus \sigma)+\mathbf{M}(\sigma)$,

(iii) $\mathbf{M}(\partial \alpha)=\mathbf{M}(\partial(\alpha \ominus \sigma))+\mathbf{M}(\partial \sigma)$.

This will be accomplished by finding points $x_{M}, \ldots, x_{N}, M, N \in \mathbf{Z}$ with $M<N$ such that

(a) $x_{i} \neq x_{j}$ unless $i=j$ or $\{i, j\}=\{M, N\}$,

(b) $\alpha\left(x_{i-1}, x_{i}\right)>0$ for $i=M+1, \ldots, N$,

(c) either (1) $x_{M}=x_{N}$ or (2) $\partial \alpha\left(x_{M}\right)<0<\partial \alpha\left(x_{N}\right)$. 
For setting $\sigma=\sum_{i=M+1}^{N}\left[x_{i-1}, x_{i}\right]$, one obtains (i), hence (ii), from (a) and (b) and (iii) from (c).

The theorem is trivial if $\alpha=0$, so choose $x_{0}$ and $x_{1}$ such that $\alpha\left(x_{0}, x_{1}\right)>0$. If $\partial \alpha\left(x_{1}\right)>0$, then set $N=1$. If $\partial \alpha\left(x_{1}\right)<0$, then we may assume that distinct points $x_{0}, \ldots, x_{k}$ have been chosen such that $\alpha\left(x_{i-1}, x_{i}\right)>0>\partial \alpha\left(x_{i}\right)$ for all $i \in$ $\{1, \ldots, k\}$. Since $0>\partial \alpha\left(x_{k}\right)=\Sigma_{y \in X} \alpha\left(y, x_{k}\right)-\alpha\left(x_{k}, y\right)$, there exists $x_{k+1}$ with the property that $\alpha\left(x_{k}, x_{k+1}\right)>0$.

If $x_{k+1}=x_{i}$ for some $i \in\{0, \ldots, k\}$, then $x_{i}, \ldots, x_{k+1}$ satisfies (a), (b), (c)(1).

If $x_{k+1} \notin\left\{x_{0}, \ldots, x_{k}\right\}$ and $\partial \alpha\left(x_{k+1}\right)>0$, then set $N=k+1$.

If $x_{k+1} \notin\left\{x_{0}, \ldots, x_{k}\right\}$ and $\partial \alpha\left(x_{k+1}\right)<0$, then continue.

Since $\{(x, y): \alpha(x, y)>0\}$ is a finite set, we either obtain the desired set or distinct points $x_{0}, \ldots, x_{N}$ such that $\alpha\left(x_{N}\right)>0$ and $\alpha\left(x_{i-1}, x_{i}\right)>0$ for all $i \in$ $\{1, \ldots, N\}$. In the latter case a similar argument using decreasing indices can be used to complete the proof.

2.3. Given a 0 -chain $\beta$ such that $\Sigma_{x \in X} \beta(x)=0$, we denote by $\mathbf{M}_{\beta}$ the number

$$
\inf \{\mathbf{M}(\alpha): \alpha \text { is a l-pchain with } \partial \alpha=\beta\} \text {. }
$$

Observe that $\mathbf{M}_{\beta}$ is finite since one can construct a 1-pchain with boundary $\beta$ by adding an appropriate number of characteristic functions $[x, y]$ where $\beta(x)<0<$ $\beta(y)$. Note also that $\mathbf{M}_{\beta}=\inf \left\{\mathbf{M}\left(\alpha_{0} \oplus \zeta\right): \zeta\right.$ is a cycle $\}$, where $\alpha_{0}$ is any 1-pchain with $\partial \alpha_{0}=\beta$.

2.4. TheOREM. If $\beta$ is a 0 -chain in $X$ such that $\Sigma \beta(x)=0$, then there exists $a$ 1-pchain $\alpha_{0}$ with $\partial \alpha_{0}=\beta$ and $\mathbf{M}\left(\alpha_{0}\right)=\mathbf{M}_{\beta}$.

Proof. For any 1-pchain $\alpha$ with $\partial \alpha=\beta$ one can find $\sigma_{i}, i=1, \ldots, l$, as in 2.2. Now define

$$
\gamma_{i}= \begin{cases}{[x, y]} & \text { if } \partial \sigma_{i}=\delta_{y}-\delta_{x}, \\ 0 & \text { if } \partial \sigma_{i}=0,\end{cases}
$$

and $\gamma=\bigoplus_{i=1}^{l} \gamma_{i}=\sum_{i=1}^{l} \gamma_{i}$. Then $\partial \gamma=\sum_{i=1}^{l} \partial \sigma_{i}=\alpha$ and by the triangle inequality

$$
\mathbf{M}(\gamma)=\sum_{i=1}^{l} \mathbf{M}\left(\gamma_{i}\right)<\sum_{i=1}^{l} \mathbf{M}\left(\sigma_{i}\right)=\mathbf{M}(\alpha) .
$$

Observing that $\gamma(x, y)>0$ implies $\beta(x)<0<\beta(y)$ we conclude

$$
\mathbf{M}_{\beta}=\inf \{\mathbf{M}(\gamma): \partial \gamma=\beta \text { and } \gamma(x, y)>0 \text { implies } \beta(x)<0<\beta(y)\} .
$$

The infimum is attained, since the latter set is finite.

2.5. TheOREM. For any positive integer $k$ and 0 -chain $\beta$ in $X$ with $\Sigma_{x \in X} \beta(x)=0$, the equality $\mathbf{M}_{\boldsymbol{k} \beta}=k \mathbf{M}_{\beta}$ holds.

PRoof. If $\alpha$ is a 1-pchain, then $\partial k \alpha=k \partial \alpha$ and $\mathbf{M}(k \alpha)=k \mathbf{M}(\alpha)$ from which $\mathbf{M}_{k \beta}<k \mathbf{M}_{\beta}$ follows.

For the reverse inequality, we suppose $\alpha$ is a 1-pchain such that $\partial \alpha=\beta$ and $\mathbf{M}(\alpha)=\mathbf{M}_{\beta}$. Given any cycle $\zeta$, we use 2.2 to decompose $\zeta$ into a sum of simple cycles $\sigma_{i}, i=1, \ldots, l$. For each $i \in\{1, \ldots, l\}$ and $(x, y) \in X \times X$, let 
Since

$$
s_{i}(x, y)= \begin{cases}-1 & \text { if }(\alpha(x, y)-\alpha(y, x))\left(\sigma_{i}(x, y)-\sigma_{i}(y, x)\right)<0 \\ 1 & \text { otherwise. }\end{cases}
$$

$$
\begin{aligned}
\alpha \oplus \sigma_{i}(x, y)+\alpha \oplus \sigma_{i}(y, x) & =\left|\alpha(x, y)-\alpha(y, x)+\sigma_{i}(x, y)-\sigma_{i}(y, x)\right| \\
& =|\alpha(x, y)-\alpha(y, x)|+s_{i}(x, y)\left|\sigma_{i}(x, y)-\sigma_{i}(y, x)\right|,
\end{aligned}
$$

we have

$$
\mathbf{M}\left(\alpha \oplus \sigma_{i}\right)=\mathbf{M}(\alpha)+\frac{1}{2} \sum_{(x, y) \in X \times X} s_{i}(x, y)\left|\sigma_{i}(x, y)-\sigma_{i}(y, x)\right| d(x, y) .
$$

By the mass minimality of $\alpha$, we conclude

$$
\sum_{(x, y) \in X \times X} s_{i}(x, y)\left|\sigma_{i}(x, y)-\sigma_{i}(y, x)\right| d(x, y)>0 .
$$

For each $(x, y) \in X \times X$ let $I(x, y)=\left\{i: s_{i}(x, y)<0\right\}$. Then

$$
\begin{aligned}
k \alpha \oplus \sum_{i=1}^{l} \sigma_{i}(x, y) & +k \alpha \oplus \sum_{i=1}^{l} \sigma_{i}(y, x) \\
= & \left|k \alpha(x, y)-k \alpha(y, x)+\sum_{i=1}^{l} \sigma_{i}(x, y)-\sigma_{i}(y, x)\right| \\
= & \mid k \alpha(x, y)-k \alpha(y, x)+\sum_{i \notin I(x, y)} \sigma_{i}(x, y)-\sigma_{i}(y, x) \\
& +\sum_{i \in I(x, y)} \sigma_{i}(x, y)-\sigma_{i}(y, x) \mid \\
= & || k \alpha(x, y)-k \alpha(y, x)\left|+\sum_{i \notin I(x, y)}\right| \sigma_{i}(x, y)-\sigma_{i}(y, x) \mid \\
= & -\sum_{i \in I(x, y)}\left|\sigma_{i}(x, y)-\sigma_{i}(y, x)\right| \mid \\
> & |k \alpha(x, y)-k \alpha(y, x)|+\sum_{i=1}^{l} s_{i}(x, y)\left|\sigma_{i}(x, y)-\sigma_{i}(y, x)\right| .
\end{aligned}
$$

Hence

$$
\begin{aligned}
\mathbf{M}(k \alpha \oplus \zeta) & >\mathbf{M}(k \alpha)+\frac{1}{2} \sum_{(x, y) \in X \times X} \sum_{i=1}^{l} s_{i}(x, y)\left|\sigma_{i}(x, y)-\sigma_{i}(y, x)\right| d(x, y) \\
& >\mathbf{M}(k \alpha)=k \mathbf{M}_{\beta} .
\end{aligned}
$$

Thus the inequality $\mathbf{M}_{k \beta}>k \mathbf{M}_{\beta}$ is established.

\section{Invariant solutions to Plateau problems.}

3.1. Let $G$ be a finite group acting on $X$ so that $d(g(x), g(y))=d(x, y)$ for all $x$, $y \in X$ and $g \in G$. Let $G(x)$ denote the orbit of $x$ and $G_{x}$ denote the isotropy 
subgroup of $G$ at $x$. Identifying orbits of $G$ to points gives the orbit space $Z$. Let $\pi$ : $X \rightarrow Z$ be the canonical projection. For each $u, v \in Z$ define

$$
d^{\prime}(u, v)=\min \left\{d(x, y): x \in \pi^{-1}(u), y \in \pi^{-1}(v)\right\} .
$$

The 0-chain $\beta$ and the 1-chain $\alpha$ are called invariant if $\beta(g x)=\beta(x)$ and $\alpha(g(x), g(y))=\alpha(x, y)$ for all $x, y \in X$.

3.2. Theorem. If $\beta$ is a G-invariant 0-chain with $\Sigma_{x \in X} \beta(x)=0$ and the order of $G$ divides $\beta(x)$ card $G(x)$ for each $x \in X$, then there exists a G-invariant 1-pchain $\alpha$ such that $\partial \alpha=\beta$ and $\mathbf{M}(\alpha)=\mathbf{M}_{\beta}$.

Proof. Let $r$ be the order of $G$. Define $\beta^{\prime}: Z \rightarrow Z$ by

$$
\beta^{\prime}(u)=\frac{1}{r} \sum_{x \in \pi^{-1}(u)} \beta(x) .
$$

Since $\beta$ is $G$-invariant and $r$ divides $\beta(x)$ card $G(x), \beta^{\prime}$ is a 0 -chain in $Z$. If $\gamma$ is a 1-pchain in $X$ with $\partial \gamma=\beta$, then one can define the 1-pchain in $Z$ by

$$
\gamma^{\prime}(u, v)=\sum_{(x, y) \in \pi^{-1}(u) \times \pi^{-1}(v)} \gamma(x, y) .
$$

Then $\partial \gamma^{\prime}=r \beta^{\prime}$ and $\mathbf{M}\left(\gamma^{\prime}\right)<\mathbf{M}(\gamma)$. Hence by $2.5, \mathbf{M}_{\beta}>\mathbf{M}_{r \beta^{\prime}}=r \mathbf{M}_{\beta^{\prime}}$.

Now choose a 1-pchain $\alpha^{\prime}$ such that $\partial \alpha^{\prime}=\beta^{\prime}$ and $\mathbf{M}\left(\alpha^{\prime}\right)=\mathbf{M}_{\beta^{\prime}}$. For each $(u, v) \in Z \times Z$ such that $\alpha^{\prime}(u, v)>0$, we find $x_{u} \in \pi^{-1}(u)$ and $y_{u, v} \in \pi^{-1}(v)$ such that $d\left(x_{u}, y_{u, v}\right)=d^{\prime}(u, v)$. Let $\alpha$ be the 1-pchain in $X$ given by

$$
\alpha=\sum_{(u, v) \in Z \times Z} \sum_{g \in G} \alpha^{\prime}(u, v)\left[g\left(x_{u}\right), g\left(y_{u, v}\right)\right] .
$$

Then

$$
\begin{aligned}
\mathbf{M}(\alpha) & =\sum_{(u, v) \in Z \times Z} \sum_{g \in G} \alpha\left(g\left(x_{u}\right), g\left(y_{u, v}\right)\right) d\left(x_{u}, y_{u, v}\right) \\
& =\sum_{(u, v) \in Z \times Z} \sum_{g \in G} \alpha^{\prime}(u, v) d^{\prime}(u, v)=r \mathbf{M}\left(\alpha^{\prime}\right),
\end{aligned}
$$

and

$$
\begin{aligned}
\partial \alpha & =\sum_{(u, v) \in Z \times Z} \sum_{g \in G} \alpha^{\prime}(u, v) \delta_{g y_{u, v}}-\sum_{(u, v) \in Z \times Z} \sum_{g \in G} \alpha^{\prime}(u, v) \delta_{g x_{u}} \\
& =\sum_{u \in Z}\left(\sum_{v \in Z} \alpha^{\prime}(v, u)-\alpha^{\prime}(u, v)\right) \sum_{g \in G} \delta_{g w_{u}} \\
& =\sum_{u \in Z} \beta^{\prime}(u) \sum_{g \in G} \delta_{g w_{u}},
\end{aligned}
$$

where $w_{u}$ is any point in $\pi^{-1}(u)$. Since $(\operatorname{card} G(x))\left(\operatorname{card} G_{x}\right)=r$, we have that

$$
\partial \alpha(x)=\beta^{\prime}(\pi(x)) \text { card } G_{x}=\beta(x) .
$$

Hence $\mathbf{M}_{\beta}<\mathbf{M}(\alpha)=r \mathbf{M}\left(\alpha^{\prime}\right)=r \mathbf{M}_{\beta^{\prime}}<\mathbf{M}_{\beta}$.

3.3. For an explanation of the terms and notation used in the following corollary, we refer the reader to [F1, pp. 670, 671]. 
COROLlary. Let $X$ be a complete Riemannian manifold and $G$ be a finite group of isometries of $X$. If $B$ is a $G$-invariant 0 -dimensional rectifiable current which is $a$ boundary and $r$ divides $\Theta(\|B\|, x)$ card $G(x)$ for all $x \in X$, then there exists $a$ $G$-invariant $T \in I_{1}(X)$ such that $\partial T=B$ and

$$
\mathbf{M}(T)=\inf \left\{\mathbf{M}(R): R \in \mathbf{I}_{1}(X) \text { and } \partial R=B\right\} .
$$

Proof. Repeat the argument of 3.2 except in the definition of $\alpha$ use $g_{\#} L\left(x_{u}, y_{u, v}\right)$ where $L\left(x_{u}, y_{u, v}\right)$ is any oriented length minimizing arc from $x_{u}$ to $y_{u, v}$.

\section{REFERENCES}

[B] D. E. Bindschadler, Invariant solutions to the oriented Plateau problem of maximal codimension, Trans. Amer. Math. Soc. 261 (1980), 439-462.

[BJ] J. E. Brothers, Invariance of solutions to invariant parametric variational problems, Trans. Amer. Math. Soc. 262 (1980), 159-179.

[F1] H. Federer, Geometric measure theory, Springer-Verlag, New York, 1969.

[F2] __ Real flat chains, cochains and variational problems, Indiana Univ. Math. J. 24 (1974), 351-407.

[L] H. B. Lawson, The equivariant Plateau problem and interior regularity, Trans. Amer. Math. Soc. 173 (1973), 231-249.

Department of Mathematics, Wayne State University, Detrott, Michigan 48202 